\title{
Gestión Económica Ambiental Del Sector Curtiembre De Ambato
}

\author{
Environmental Management Economic Sector Ambato Tannery
}

Mery Esperanza Ruiz Guajala1, César Medardo Mayorga Abril2, Luis Marcelo Mantilla Falcón ${ }^{3}$, Patricia Alexandra López Chalán ${ }^{4}$

1. Mery Esperanza Ruiz Guajala, Economista, Magíster en Costos y Gestión Financiera. Docente en la Facultad de Contabilidad y Auditoría de la Universidad Técnica de Ambato. meryeruiz@uta.edu.ec

\footnotetext{
2. César Medardo Mayorga Abril, Doctor en Contabilidad y Auditoría, Magíster en Costos y Gestión Financiera. Docente en la Facultad de Contabilidad y Auditoría de la Universidad Técnica de Ambato. cesarmmayorga@uta.edu.ec
} 3. Luis Marcelo Mantilla Falcón: Doctor en Ciencias de la Educación, Magíster en Docencia Universitaria y Administración Educativa. Docente
en la Facultad de Contabilidad y Auditoría de la Universidad Técnica de Ambato. luismmantilla@uta.edu.ec

4. Patricia Alexandra López Chalán: Economista. Docente en el Sistema de Nivelación de la Facultad de Contabilidad y Auditoría de la Universidad Técnica de Ambato. patty_lopez1031@yahoo.com

\section{Resumen}

En América Latina el sector de las curtiembres, aunque es generador de empleo y riqueza, tiene serios problemas ambientales producidos por dicha industria. Las curtiembres ocasionan contaminación especialmente en el agua, lo que provoca serias dificultades ambientales, intoxicando la biodiversidad por la descarga de altas concentraciones de materia orgánica, sulfuros, cromos, sulfatos, nitratos, carbonatos, grasas y aceites, entre otros, la realidad ecuatoriana y muy especialmente de la ciudad de Ambato, no es diferente. Esta investigación tiene como objetivo principal contrastar la gestión ambiental en el sector curtiembre de la ciudad de Ambato, Ecuador, entre los años 2011 y 2015 y caracterizar la conducta empresarial de dicho sector. La recolección de la información fue mediante encuestas a los propietarios y/o administradores de las curtiembres en dos momentos. El resultado más importante evidencia un salto cualitativo y cuantitativo en el cuidado y protección del medio ambiente en cumplimiento de las normativas vigentes y como un compromiso socialmente responsable del sector empresarial.

Palabras clave: Gestión ambiental, curtiembres, economía, medioambiente, contaminación ambiental. 


\begin{abstract}
In Latin America the tannery sector, although it is generating employment and wealth, has serious environmental problems caused by the industry. Tanneries cause contamination especially water, causing serious environmental problems, biodiversity poisoning by the discharge of high concentrations of organic matter, sulphides, chrome, sulfates, nitrates, carbonates, fats and oils, among others, the Ecuadorian reality especially in the city of Ambato, is no different. This research has as main objective to compare the environmental management in the tannery city of Ambato, Ecuador sector between 2011 and 2015 and characterize the behavior of the business sector. The information was collected through surveys of owners and / or managers of the tanneries in two stages. The most important result shows a qualitative and quantitative leap in the care and protection of the environment in compliance with the regulations and as a socially responsible business engagement.
\end{abstract}

Keywords: Environmental management, tanneries, economy, environment, environmental contamination.

\title{
1. Introdution
}

En la actualidad se vive en un mundo en donde las exigencias del mercado nacional e internacional, en cuanto a la competitividad, en la calidad del producto, precios y condiciones ambientales han generado nuevos desafíos de sustentabilidad para la industria de las curtiembres.

En América Latina el sector de las curtiembres, aunque es generador de empleo y riqueza, tiene serios problemas ambientales producidos por dicha industria. Las curtiembres ocasionan contaminación especialmente en el agua, lo que provoca serias dificultades ambientales, intoxicando la biodiversidad, por la descarga de altas concentraciones de materia orgánica, sulfuros, cromos, sulfatos, nitratos, carbonatos, grasas y aceites, entre otros.
En Argentina, en 1998 se informó de niveles muy altos de contaminación por cromo, que superaban varias veces los permisibles, en 24 pobladores cercanos a dos curtiembres localizadas en Santa Fe, además de la aparición de 12 casos de leucemia en estos pobladores. La característica común de estas dos industrias es una inadecuada disposición en el ambiente de los residuos (Téllez, Carvajal, \& Gaitán, 2004).

En Colombia se produjo el cierre de 26 curtiembres debido que no contaban con las normas ambientales necesarias, por arrojar los desechos químicos a los ríos aledaños (Radio Santa Fe, 2008).

En el Perú, los métodos tradicionales de producción de cuero, consumen entre el 60 al 80\% 
del cromo utilizado para el proceso de curtido, generando aguas residuales con altas concentraciones de cromo total que deben ser tratadas para poder cumplir con los Valores Máximos Admisibles (VMA) (Córdova, Vargas, Cesare, Flores, \& Visitación, 2014).

En el Ecuador la industria del curtido de pieles se encuentra estrechamente ligada a dos importantes sectores productivos del país: la del sacrificio de animales, para la adquisición de pieles y a la fabricación de calzado, a quien provee de materia prima, en calidad de cueros terminados.

Las provincias con una mayor producción de pieles curtidas son: Tungurahua 76\%, Imbabura 13\%, Azuay y Cotopaxi 4\% cada uno, otros 3\% (Ministerio Coordinador de la Producción Empleo y Competitividad, 2010), lo que demuestra que a nivel nacional, la mayor producción se concentra en las provincias de la Sierra, mientras que en el resto del país la elaboración de cuero se da en una menor proporción.

En la provincia de Cotopaxi existe un avanzado deterioro ambiental en el Río Cutuchi y el Canal de Riego Latacunga - Salcedo - Ambato, causado por la descarga diaria de desechos tóxicos que son arrojados a dichos afluentes provenientes de las curtiembres, camales e industrias.

Todos los daños ocasionados al medio ambiente, por causa de las curtiembres, demuestran que ninguna de estas industrias cuenta con la infraestructura ni un sistemas de costos ambientales, es decir, no destinan recursos económicos para ayudar a la conservación del medio ambiente ni para remediar los daños causados a los ecosistema.

En lo que se refiere al ámbito social y económico ecuatoriano, el sector cuero generaba 100.000 puestos en curtiembres, 330.000 en producción de calzado, 80.000 personas en la fabricación de hormas, plantas y maquinarias y 290.000 en comercialización, dando un total de 800.000 puestos de trabajo; contribuyendo tanto al desarrollo social y económico del país (Ministerio Coordinador de la Producción Empleo y Competitividad, 2010), por lo que este sector necesita ser impulsado de mejor manera dado que genera una gran cantidad de fuentes de empleo.

Para la provincia de Tungurahua, la producción de cuero es parte de una cadena productiva que comienza con los proveedores de materia prima (pieles), curtiembres, el sector productor de calzado, los proveedores de accesorios y los centros de expendio o comercialización.

Según datos de la Asociación Nacional de Curtidores Ecuatorianos - ANCE, existen 1.770 talleres artesanales que se dedican a la confección de cuero y a la zapatería, lo que representa el 75,58\% de la actividad artesanal en el Ecuador. Sus principales talleres se localizan en los cantones de 
Ambato, Baños, Cevallos (Ministerio Coordinador de la Producción Empleo y Competitividad, 2010).

El proceso de producción de la curtiembre genera apreciables efectos negativos al medio ambiente en los componentes aire: olores, material particulado, gases y humo. En el componente agua, se aprecian efectos por demanda (extracción) de agua de las fuentes naturales (ríos, quebradas, etcétera), así como por la contaminación de ríos y quebradas a consecuencia de la disposición de aguas residuales derivadas del proceso productivo (Vásquez \& Gonzales, 2009) .

La producción de cueros con diferentes grados de terminado, se realiza en áreas sobre las que convergen varios factores que le sean favorables, entre ellos: fácil acceso al agua, disponibilidad y costo de materia prima y mano de obra, así como

En el caso de la curtiembre ambateña, la cadena de valor, está integrada por la ganadería (faenamiento), a continuación, las pieles son procesadas por las curtiembres, las mismas que obtienen los cueros, que sirven para la elaboración de calzado y otros productos a través de la oportunidades de transporte y comercialización (Corredor, 2006).

Para los mismos autores, las aguas residuales, generadas en la producción del cuero, generalmente, son vertidas a un sistema de alcantarillado público, a un río u otro afluente hídrico. Estos vertimientos presentan alta concentración de carga orgánica, debido a que incluyen sustancias, tales como estiércol, sangre, barro, sal y microorganismos; restos de sebo, residuos sólidos (carnaza), pelo, sulfuros sólidos, nitrógeno, sólidos en suspensión (proteínas disueltas), residuos alcalinos, residuos ácidos (algunos con presencia de $\mathrm{Cr}^{3+}$ ), cloruros, sulfatos, colorantes (naturales, artificiales y sintéticos), ácido fórmico y otras sustancias químicas (Vásquez \& Gonzales, 2009).

marroquinería. La cadena de valor del cuero, calzado y marroquinería tiene el desafío de avanzar en la industrialización de la ruralidad, es decir, en el desarrollo de polos productivos cercanos a las fuentes de recursos naturales que permita un crecimiento federal y acompañe el corrimiento de la frontera ganadera (Ministerio de Industria Presidencia de la Nación, 2012). 
Resumen de los procesos de curtido

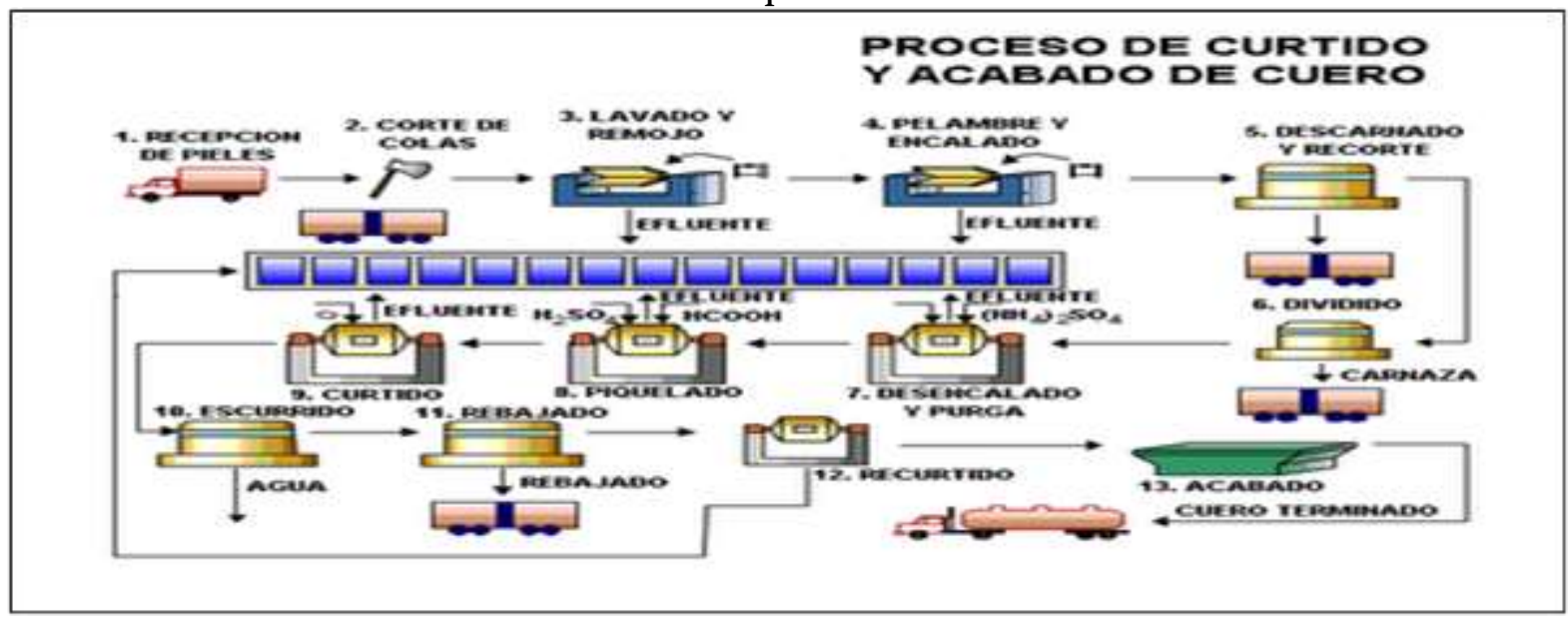

Figura. 1. Proceso de curtido y acabado de cuero

Fuente: http://www.tecnologiaslimpias.org/html/central/323101/323101_ee.htm

De manera resumida se señala que se diferencia cuatro etapas bien marcadas: La etapa de la ribera, que es la recepción de pieles, salado, remojo y/o lavado, pelambre, descarnado y dividido. La etapa de curtido, que se encarga del desencale y purgado, lavado, piquelado curtido y basificado; al culminar esta etapa el cuero se denomina wet blue. La etapa de post-curtido, consiste en el escurrido, rebajado, neutralizado, lavado, recurtido, teñido y engrasado, lavado y secado. Finalmente la etapa de acabado, y consiste en el acondicionado, palizonado y tesado, pintado, batanado y planchado y se obtiene el cuero acabado (Córdova-Bravo, Vargas-Parker, Cesare-Coral, \& Visitación-Figueroa, 2014).

De acuerdo a su grado de tecnificación, las curtiembres ambateñas, obtienen sus productos finales con, aparentemente, menos procesos que los detallados en el esquema anterior, porque algunas de ellas actúan de manera artesanal.

\section{Aspectos e impactos ambientales generados por las curtiembres}

Un aspecto es un elemento de las actividades curtidoras que tienen o pueden tener una implicación positiva o negativa. El impacto es una alteración al medio ambiente que puede ser positiva o negativa $y$, por lo tanto, puede perjudicar o beneficiar al mismo (Centro Nacional de Producción Más Limpia, 2004), los impactos que generan las curtiembres se los observa en el agua, suelo y aire. Entre los aspectos e impactos, que generalmente son similares en todas las industrias curtidoras se tiene: 
Cuadro 1. Aspectos e impactos ambientales de las curtiembres.

\begin{tabular}{ll}
\hline Aspecto ambiental & Impacto ambiental \\
\hline Consumo de insumos & Uso de recursos \\
Consumo de energía eléctrica y térmica & Uso de recursos \\
Consumo de agua & Uso de recursos \\
Generación de emisiones atmosféricas & - VOC’S del acabado. \\
& - Ácido sulfhídrico del proceso de encalado. \\
& - Amoniaco del proceso de desencalado. \\
& - Gases de invernadero producidos por las calderas, generadores \\
& eléctricos y otros equipos que consumen combustible. \\
Efluentes de aguas residuales & Por DBO en los procesos de ribera (remojo, depilado, encalado, \\
& desencalado, rendido). \\
& - Salinidad: Remojo. \\
& - Amoniaco: Desencalado. \\
Desechos sólidos & - Materia orgánica putrescible o residuos de piel. \\
\hline
\end{tabular}

Fuente: Centro Nacional de Producción Más Limpia, 2004

Las industrias curtidoras de alto impacto generan efectos nocivos por las descargas líquidas no domésticas, emisión de combustión, emisiones de procesos y ruido, residuos sólidos (El Heraldo, 2015).

\section{Gestión Ambiental}

La dimensión de la responsabilidad en la protección del medio ambiente — como una labor compartida entre la sociedad, el gobierno y las empresas - es un argumento del debate entre la sustentabilidad y el beneficio económico que se propone, desde la revisión de políticas económicas nacionales hasta la instrumentación de beneficios financieros que ayuden y motiven a las organizaciones a enfrentar los diferentes niveles de compromiso con su entorno natural y lograr así, un beneficio social. Teóricamente, la gestión medioambiental es vista como una serie de actividades y metas insertadas en los procesos de producción que implantan valores tales como el respeto y cuidado del medio ambiente al disminuir riesgos por insumos, residuos o procesos (de la Rosa Leal, 2007). Todo esto está encaminado a lograr una adecuada gestión ambiental en las empresas curtidoras.

La Gestión ambiental, es el conjunto de acciones encaminadas a lograr la maximización del cuidado ambiental, considerado como un activo intangible en el marco de la Responsabilidad Social y compromiso con la protección del entorno en su área de influencia. de la Rosa Leal (2007) considera que es necesario proponer y crear nuevas unidades administrativas dentro de la empresa para atender la ecología de la organización para la interacción entre ésta y la naturaleza, esta visión considera el enfoque de sistemas, el énfasis en la cultura interna y el uso de la tecnología para establecer y desarrollar una gestión medioambiental en la organización. El resultado que establece menciona 
que es necesario establecer medidas preventivas y correctivas y concluye que el compromiso ambiental de la planta es de naturaleza legal.

\section{Metodología}

La presente investigación es de carácter no experimental, descriptiva, cualicuantitativa, longitudinal con toma de datos en dos temporalidades: la primera vez en el año 2011 a 40 curtiembres y la segunda en el 2015 a 20 de la ciudad de Ambato, Ecuador. Se aplicó muestreo no probabilístico intencional, los datos fueron recogidos mediante una encuesta directa a los propietarios $y / o$ administradores de las curtiembres considerando variables como: disposición y eliminación de aguas servidas, licencia ambiental, presupuestos, entre otras.

\section{Resultados y Discusión}

La participación del sector curtiembre, para este estudio, se divide en tres: curtidurías con el 60\% y son aquellas que tiene una estructura bien definida y los procesos son tecnificados. El 30\% pertenecen a talleres de manufactura artesanal y finalmente el $10 \%$ son empresas legalmente constituidas.

Geográficamente la distribución de las curtidurías se encuentra en zonas suburbanas y rurales del cantón Ambato, como se detalla en la figura 2.

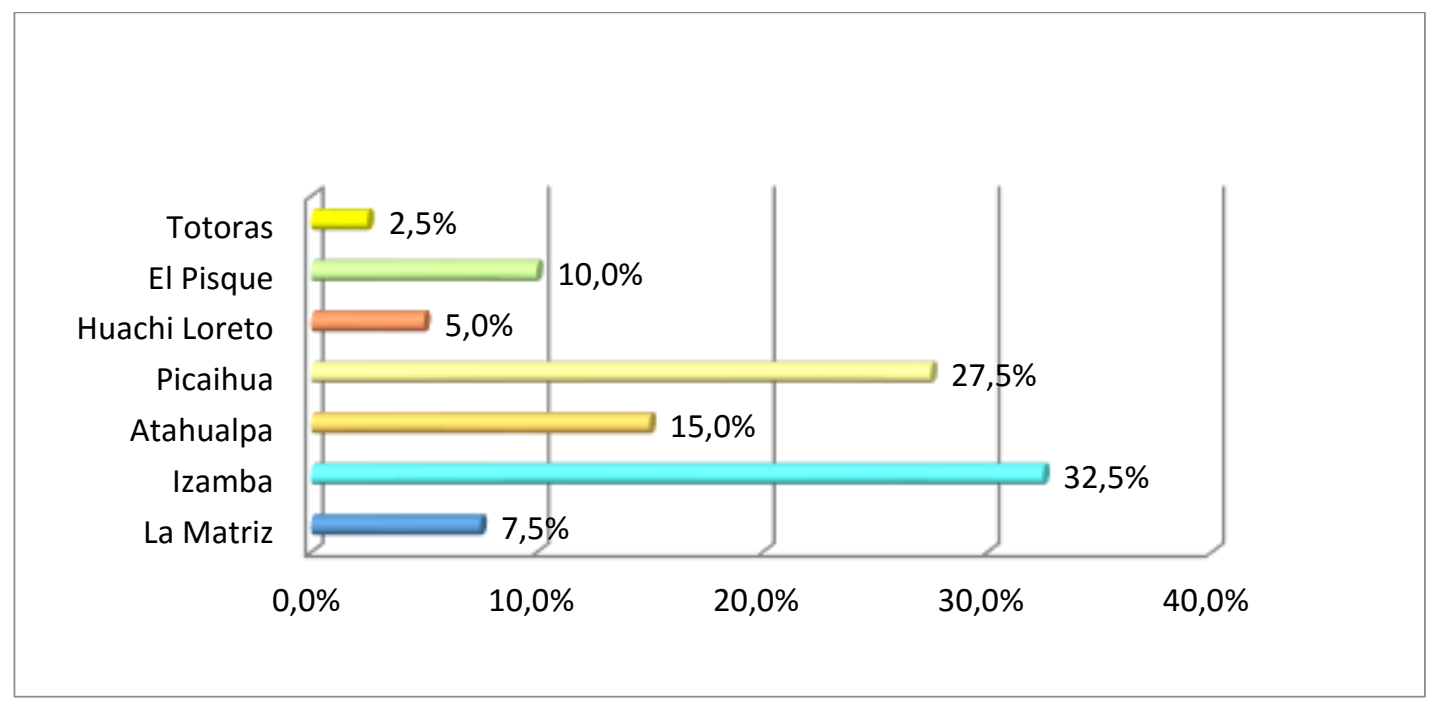

Figura 2. Distribución del sector curtiembre.

Las parroquias urbano-rurales de Izamba y Picaihua suman el 60\% del sector curtidor del cantón por las facilidades de evacuación de desechos hacia el río que pasa por el sector. 
El estudio evidencia datos muy significativos en función del tratamiento que se da a las aguas residuales, para el 2011 sólo el 5\% de las curtidurías ejecutaban algún proceso de tratamiento de aguas; mientras que al 2015, los porcentajes se invierten. El $85 \%$ hace un tratamiento primario, el 5\% completo, el 5\% biológico y el 5\% ningún proceso.

Los procesos de eliminación de las aguas se describen en la tabla 1.

Tabla 1. Eliminación de aguas residuales

\begin{tabular}{lll}
\hline Opciones & Porcentajes & \\
\cline { 2 - 3 } Alcantarillado & 2011 & 62 \\
Ríos & 82 & 8 \\
Afluentes de agua & 8 & 5 \\
Tierra & 5 & 10 \\
Otros (planta de tratamiento) & 5 & 15 \\
TOTAL & 0 & 100 \\
\hline
\end{tabular}

Fuente: Encuesta

La información evidencia que la práctica más recurrente de las curtiembres es eliminar sus desechos hacia el alcantarillado público puesto que esto representa un gran ahorro económico para sus empresas a costa del alto perjuicio al medio ambiente en vista que todos sus residuos desembocan en el río del sector geográfico.

La frecuencia de eliminación de los desechos de manera diaria pasó, en los años de estudio, del 63\% en el 2011 al 50\% en el 2015, porque algunas empresas construyeron sus propias plantas de tratamiento de aguas.

Para en 2011 solo el 12,5\% de las empresas consideraban costos ambientales en sus presupuestos, al 2015 se alcanzó el 65\%, demostrando que se ha dado mayor importancia al factor ambiental.

La variación por efectos de contar con licencia ambiental ha sido muy significativa para este sector, pasando del 12,5\% en el año 2011 al 80\% para el 2015. En función de lo que dispone el Texto Unificado de la Legislación Secundaria (TULAS), en especial el libro II y VI.

Bajo el escenario anterior, muchas curtidurías modificaron su accionar en función de las exigencias reglamentarias propuestas por las autoridades y organismos competentes, tanto es así que para el 2015, el 60\% de las empresas cuentan con el Manual de la ficha Ambiental; el 95\% cuenta con Estudios de Impactos 
Ambientales (EIA), un significativo porcentaje $(35 \%)$ ha ejecutado, al menos una vez una auditoría ambiental. El 70\% cuenta con Planes de Manejo Ambiental, los mismos que nacen como consecuencia de los informes de las auditorias.

El $80 \%$ porciento aplica la política de "Buenas Prácticas Ambientales", de otro lado, solo el 45\% cumple con el pago del impuesto ambiental, exigido por la alcaldía de la ciudad.

Los empresarios del sector curtiembre exponen algunas razones por las que es importante implementar estrategias de desarrollo sustentable. Sus datos se evidencian en la tabla 2.

Tabla 2. Razones para establecer estrategias de desarrollo sustentable.

\begin{tabular}{ll}
\hline Estrategias & Porcentaje \\
\hline Aumentar la reputación de la marca o industria & $0 \%$ \\
Captar clientes & $10 \%$ \\
Diferenciar productos & $10 \%$ \\
Cumplir con obligaciones legales y regulaciones & $60 \%$ \\
Mejorar condiciones laborales & \\
Conciencia empresarial & $15 \%$ \\
Total & $5 \%$ \\
\hline
\end{tabular}

Fuente: Encuesta

\section{Conclusiones}

-Se evidencia un cambio positivo significativo en la conducta del empresario del sector curtiembre de la ciudad de Ambato tomando en consideración el componente ambiental.

-Se genera un claro compromiso de responsabilidad ambiental al implementar los requerimientos exigidos por la normatividad vigente (TULAS) y cumplir con los estándares ambientalmente permitidos.

-Habido un salto cualitativo y cuantitativo en el cuidado y protección del medioambiente pero aún falta mucho por hacer en los niveles gerenciales y de quienes toman decisiones en el sector.

\section{Bibliografía}

Centro Nacional de Producción Más Limpia. (2004). Gestión Ambiental en la Industria de Curtiembres. Colombia: Centro Nacional de Producción Más Limpia y Tecnologías Ambientales, CNPMLTA. 
Córdova, H., Vargas, R., Cesare, M., Flores, L., \& Visitación, L. (2014). Tratamiento de las aguas residuales del proceso de curtido tradicional y alternativo que utiliza acomplejantes de cromo. Revista de la Sociedad Química del Perú, 183.

Córdova-Bravo, H. M., Vargas-Parker, R., CesareCoral, M. F.-d., \& Visitación-Figueroa, L. (2014). Tratamiento de las aguas residuales del proceso de curtido tradicional y alternativo que utiliza acomplejantes de cromo. Revista de la Sociedad Química del Perú, 183-191.

Corredor, J. L. (agosto-diciembre de 2006). El residuo líquido de las curtiembres estudio de caso: cuenca alta del Río Bogotá. Ciencia e Ingeniería Neogranadina, 16(2), 14-28.

de la Rosa Leal, M. E. (2007). La responsabilidad y la gestión medioambiental de la industria maquiladora. Un estudio de caso. Contaduría y Administración, 83-108.

El Heraldo. (27 de Julio de 2015). El Heraldo. Obtenido de El Heraldo web site: www.elheraldo.com.ec

Ministerio Coordinador de la Producción Empleo y Competitividad. (2010). Estudio Técnico para la identificación de la Politica Comercial necesaria para que el sector del cuero y calzado del Ecuador se desarrolle y aumente su participación mundial en las exportaciones. Quito: MCPEC.

Ministerio de Industria Presidencia de la Nación. (2012). Cadena de valor del cuero, calzado y marroquinería Plan estratégico industrial 2020. Buenos Aires: Ministerio de Industria Presidencia de la Nación.

Ministerio de Industrias y Productividad. (2013). Sector curtiembre creció un 8,6\% entre 2011 y 2012. Ambato: Ministerio de Industrias y Productividad.

Radio Santa Fe. (22 de Diciembre de 2008). Cierran 26 curtiembres por contaminación en el sur de Bogotá. Obtenido de Radio Santa Fe: www.radiosantafe.com

Téllez, J., Carvajal, R., \& Gaitán, A. (2004). Aspectos toxicológicos relacionados con la utilización del cromo en el proceso productivo de curtiembres. Rev Fac Med Univ Nac Colombia, 52.

Vásquez, J. A., \& Gonzales, D. P. (noviembre de 2009). Metodología para implementar un modelo de responsabilidad social empresarial (RSE) en la industria de la curtiembre en Colombia. Contabilidad y Negocios, 4(8), 49-56. 